\title{
New avenues to treatment of liver cirrhosis
}

\author{
WAN XueShuai \& ZHAO HaiTao* \\ Department of Liver Surgery, Peking Union Medical College Hospital, Chinese Academy of Medical Sciences and Peking Union Medical \\ College, Beijing 100730, China
}

Received August 20, 2014; accepted September 2, 2014; published online September 16, 2014

Citation: Wan XS, Zhao HT. New avenues to treatment of liver cirrhosis. Sci China Life Sci, 2014, 57: 1049-1050, doi: 10.1007/s11427-014-4751-x

Liver cirrhosis is the pathologic end stage of multiple liver diseases. The major complications of liver cirrhosis, such as hepatic encephalopathy, spontaneous bacterial peritonitis and esophageal variceal bleeding are characterized by remarkable changes of the gut microbiota, which indicates that enteric dysbiosis might play an important role in the progression of liver cirrhosis [1,2]. The human gastrointestinal tract hosts 500-1500 different bacteria species containing nearly $10^{14}$ bacteria, which is 10 -fold larger than that of human cells $[3,4]$. However, their phylogenetic and functional composition changes in liver cirrhosis are still largely unknown.

Using quantitative metagenomics analysis developed by metagenomics of the Human Intestinal Tract [6], Prof. Li and her team built the world's first reference gene set of gut microbiota in liver cirrhosis by studying stool samples from 98 patients and 83 healthy control individuals. Of the 2.69 million genes in this liver cirrhosis catalogue, $36.1 \%$ of which are novel, indicating that more unknown genes could be identified by inclusion of more subjects. They also discovered the compositional changes in gut microbiota related to liver cirrhosis. At the phylum level, Bacteroidetes was significantly decreased in the liver cirrhosis group, while Proteobacteria and Fusobacteria were increased. At the genus level, patients with liver cirrhosis had fewer Bacteroides, but higher levels to Veillonella, Streptococcus, Clostridium and Prevotella. To investigate the microbial genes associated with liver cirrhosis, Prof. Li's group identified a total of 75245 genes and further grouped them into

*Corresponding author (email: zhaoht@pumch.cn)
66 metagenomic species. Interestingly, Faecalibacterium prausnitzii and Coprococcus comes were enriched in healthy controls and they had the ability to fight inflammation and produce butyrate, respectively. These findings will facilitate therapeutic attempts to correct the major dysbioses in gut microbiota and therefore open new avenues to treatment of liver cirrhosis.

When the origin of species enriched in patient groups were explored using HOMD and GOLD databases [6], surprisingly, 54\% of them were found to be of buccal origin, indicating that a major change of gut microbiota in the patients with liver cirrhosis might be the consequence of a massive invasion of the gut by oral bacterial species.

The functional role of the gut microbiota in liver cirrhosis was elucidated by identifying their orthologues [6]. At the module or pathway level, the liver-cirrhosis-associated markers included various pathophysiologic processes in cirrhosis complications, especially hepatic encephalopathy. To translate their research findings to clinical practice, they selected 15 optimal gene markers and proposed a patient discrimination index (PDI), which could discriminate patients with liver cirrhosis from healthy individuals, with a high specificity.

The study [6] provided us a more profound understanding of the gut microbiota in liver cirrhosis. However, as we know, hepatitis B virus infection is one of the leading causes to liver cirrhosis in China and other Asian countries, while alcohol-related cirrhosis is a major cause of morbidity and mortality in Western countries. In Prof. Li's previous study, they found the fecal microbial communities between HBV-related and alcohol-related cirrhosis was similar and 
concluded that the composition changes between different fecal microbiota were mostly due to cirrhosis [5]. Recently, Bajaj et al. found a different pattern of dysbiosis in alcoholic cirrhosis with higher Enterobacteriaceae and endotoxemia compared to non-alcoholic patients [1]. Therefore, it seemed to be necessary to compare the gut microbiota between HBV-related and alcohol-related cirrhosis with relatively larger sample size in future studies.

Taken together, this is an important study demonstrating the alterations of the human gut microbiome in liver cirrhosis. Understanding the exact compositional changes will greatly enhance our ability to modulate the gut microbiota and treat liver cirrhosis.

1 Bajaj JS, Heuman DM, Hylemon PB, Sanyal AJ, White MB, Monteith P, Noble NA, Unser AB, Daita K, Fisher AR, Sikaroodi M,
Gillevet PM. Altered profile of human gut microbiome is associated with cirrhosis and its complications. J Hepatol, 2014, 60: 940-947

2 Bajaj JS, Ridlon JM, Hylemon PB, Thacker LR, Heuman DM, Smith S, Sikaroodi M, Gillevet PM. Linkage of gut microbiome with cognition in hepatic encephalopathy. Am J Physiol Gastrointest Liver Physiol, 2012, 302: G168-175

3 Henao-Mejia J, Elinav E, Thaiss CA, Licona-Limon P, Flavell RA. Role of the intestinal microbiome in liver disease. J Autoimmun, 2013, 46: 66-73

4 Goel A, Gupta M, Aggarwal R. Gut microbiota and liver disease. J Gastroen Hepatol, 2014, 29: 1139-1148

5 Chen Y, Yang F, Lu H, Wang B, Chen Y, Lei D, Wang Y, Zhu B, Li L. Characterization of fecal microbial communities in patients with liver cirrhosis. Hepatology, 2011, 54: 562-572

6 Qin N, Yang F, Li A, Prifti E, Chen Y, Shao L, Guo J, Le Chatelier E, Yao J, Wu L, Zhou J, Ni S, Liu L, Pons N, Batto JM, Kennedy SP, Leonard P, Yuan C, Ding W, Chen Y, Hu X, Zheng B, Qian G, Xu W, Ehrlich SD, Zheng S, Li L. Alterations of the human gut microbiome in liver cirrhosis. Nature advance online publication 23 July 2014; doi: 10.1038 /nature 13568

Open Access This article is distributed under the terms of the Creative Commons Attribution License which permits any use, distribution, and reproduction in any medium, provided the original author(s) and source are credited. 